\title{
Review Article \\ Prognostic Value of Pretreatment Albumin-to-Alkaline Phosphatase Ratio in Cancer: A Meta-Analysis
}

\author{
Hailun Xie, ${ }^{1}$ Lishuang Wei, ${ }^{2}$ Shuangyi Tang, ${ }^{3}$ and Jialiang Gan $\mathbb{D i D}^{1}$ \\ ${ }^{1}$ Department of Colorectal and Anal Surgery, The First Affiliated Hospital, Guangxi Medical University, Nanning, Guangxi, China \\ ${ }^{2}$ Geriatric Respiratory Disease Ward, The First Affiliated Hospital, Guangxi Medical University, Nanning, Guangxi, China \\ ${ }^{3}$ Department of Pharmacy, The First Affiliated Hospital, Guangxi Medical University, Nanning, Guangxi, China
}

Correspondence should be addressed to Jialiang Gan; gj15172@163.com

Received 3 October 2020; Revised 19 November 2020; Accepted 1 December 2020; Published 10 December 2020

Academic Editor: Musthafa M. Essa

Copyright (c) 2020 Hailun Xie et al. This is an open access article distributed under the Creative Commons Attribution License, which permits unrestricted use, distribution, and reproduction in any medium, provided the original work is properly cited.

Background. Recently, it has been reported that the pretreatment albumin-to-alkaline phosphatase ratio (AAPR) is related to the prognosis of various cancers. The purpose of this systematic review and meta-analysis was to explore the prognostic value of pretreatment AAPR on clinical outcomes in cancer. Methods. PubMed, Web of Science, Cochrane Library, and Embase were systematically searched for relevant research before May 2020. Stata 12 was utilized to extract the data and the characteristics of each study and to generate a pooled hazard ratio (HR) and 95\% confidence interval (CI) to assess the relationship between pretreatment AAPR and survival outcomes. Results. We included 16 eligible published articles involving 5,716 patients. We found that low pretreatment AAPR was associated with poor overall survival (HR $=2.12,95 \% \mathrm{CI}: 1.80-2.50, P<0.001$ ), cancerspecific survival ( $\mathrm{HR}=2.89,95 \% \mathrm{CI}: 1.46-5.71, P<0.001)$, disease-free survival ( $\mathrm{HR}=1.91,95 \% \mathrm{CI}: 1.43-2.53, \mathrm{P}<0.001)$, and progression-free survival ( $\mathrm{HR}=1.93,95 \% \mathrm{CI}: 1.49-2.52, \mathrm{P}<0.001)$. However, there was no statistical relationship between pretreatment AAPR and recurrence-free survival, distant-metastasis-free survival, or locoregional relapse-free survival. The correlation between pretreatment AAPR and overall survival did not change significantly when possible confounders were stratified. The sensitivity analysis showed that this study was reliable. Conclusions. Low pretreatment AAPR was significantly associated with adverse clinical outcomes of cancer. Pretreatment AAPR could be a valuable noninvasive prognostic indicator for cancer.

\section{Introduction}

According to the World Health Organization, cancer-related deaths accounted for approximately one-sixth of total global deaths in 2015. The latest Global Cancer Statistics estimated 18.1 million new cancer cases and 9.6 million cancer deaths worldwide in 2018. Asia accounts for nearly half of new cancer cases and nearly $70 \%$ of cancer deaths. In China, the incidence and death rate of cancer ranks first in the world, with about 3.804 million new cases and 2.296 million deaths [1]. Cancer remains one of the leading causes of death worldwide and is a major obstacle to increasing life expectancy in every country in the 21st century [2]. Therefore, it is imperative to find more simple and convenient biological indicators to predict the prognosis of cancer, especially in the Asian population.
In recent years, a large number of studies have reported that inflammation and nutritional status play important roles in the onset, development, and therapeutic response of cancer and are significant factors affecting the clinical outcome of cancer patients [3-5]. In clinical practice, inflammatory and nutritional status is usually determined by routine blood and biochemical markers. Many indicators based on inflammation and nutritional status have been used to predict the prognosis of cancer, including the Glasgow prognostic score [6], modified Glasgow prognostic score [7], C-reactive protein-albumin ratio [8], and Geriatric Nutritional Risk Index [9]. Albuminto-alkaline phosphatase ratio (AAPR), as a new inflammatory and nutrition-related indicator, has attracted increasing attention. Recent evidence confirms that pretreatment AAPR is associated with adverse outcomes in patients with 


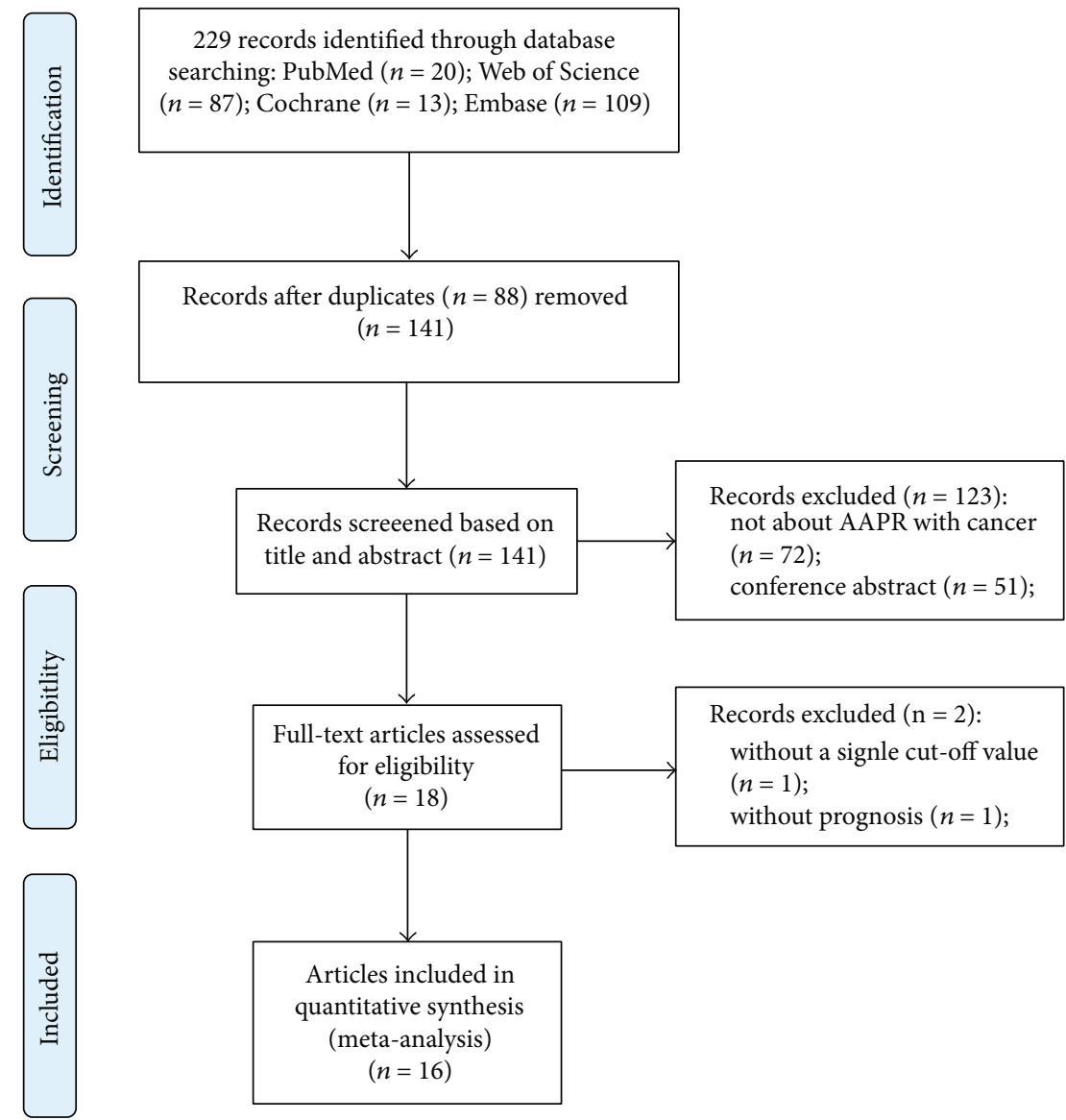

FiguRE 1: The flow chart of the literature selection.

various cancers, including hepatocellular carcinoma (HCC) [10], nasopharyngeal carcinoma (NPC) [11], non-smallcell lung cancer (NSCLC) [12], upper tract urothelial carcinoma (UTUC) [13], and breast cancer [14]. However, due to the differences in study design and sample size of these studies, there are still some contrary results. The association between pretreatment AAPR and cancer outcomes remains controversial [15]. The prognostic value of pretreatment AAPR in cancer has rarely been systematically investigated.

Therefore, it is necessary to evaluate the relationship between pretreatment AAPR and cancer outcomes based on available evidence. We performed a systematic review and meta-analysis to investigate the prognostic value of pretreatment AAPR in cancer patients.

\section{Methods}

2.1. Search Strategy and Study Selection. We performed a systematic literature search on PubMed, Web of Science, Cochrane Library, and Embase, with a cutoff date of May 10, 2020. The search strategy combined main keywords and free words and used a combination of the following search terms: ("Albumin-to-Alkaline phosphatase" OR "Albumin/Alkaline phosphatase" OR "ALB/ALP" OR "ALB-ALP" OR “AAPR") AND ("neoplasms" OR “carcinoma” OR "leukemia" OR "lymphoma”). To avoid duplicate studies, we also examined all authors and organizations of the included articles and assessed the recruitment period and number of patients in each article. Additionally, we conducted a manual review of references to identify potentially relevant studies from the retrieved publications. The registration number in the international prospective register of systematic reviews (PROSPERO) was CRD42020206902.

2.2. Inclusion and Exclusion Criteria. Based on the PICOS criteria, we strictly screened the eligible studies for inclusion in the meta-analysis: (1) population-patients with cancer; (2) intervention-patients receiving surgery, chemoradiotherapy, or nonstandard treatment; (3) comparison-cancer patients with low AAPR and high AAPR; (4) outcomes-primary outcome: overall survival (OS), secondary outcome: disease-free survival (DFS), cancer-specific survival (CSS), distant-metastasis-free survival (DMFS), locoregional relapse-free survival (LRRFS), progression-free survival (PFS), and recurrence-free survival (RFS); and (5) study design-comparative cohort study (retrospective study or prospective study). The exclusion criteria were as follows: (1) population-research on patients without cancer; (2) intervention-research on the value of AAPR after treatment; (3) comparison-grouping for AAPR is $\geq 3$ groups, or hazard ratio (HR) and $95 \%$ confidence interval (CI) could not be extracted; (4) outcomes-studies without primary or secondary results; and (5) study design-one-arm 


\begin{tabular}{|c|c|c|c|c|c|c|c|c|c|c|c|c|c|c|c|c|c|c|c|}
\hline$z$ & $\infty$ & $\infty$ & $\infty$ & $\infty$ & $\infty$ & $\infty$ & $\infty$ & $\infty$ & $\infty$ & $\infty$ & 6 & $\infty$ & 0 & $\infty$ & $\infty$ & $\infty$ & $\wedge$ & $\begin{array}{lll}0 & \infty\end{array}$ & \\
\hline 齿 & $\Sigma$ & $\Sigma$ & $\Sigma$ & $\Sigma$ & $\Sigma$ & $\Sigma$ & $\Sigma$ & $\Sigma$ & $\Sigma$ & $\Sigma$ & $D$ & $\Sigma$ & D & $\Sigma$ & $\Sigma$ & $\Sigma$ & & P & \\
\hline$\pi$ & Fy & $\aleph_{0}^{\infty}$ & 帣 & 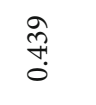 & 氶 & 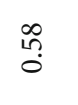 & 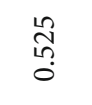 & $\begin{array}{l}0 \\
\text { o } \\
\text { क्ष } \\
0\end{array}$ & 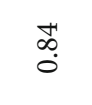 & in & $\stackrel{\overrightarrow{0}}{\circ}$ & ले & के & F & : & 苛 & $\begin{array}{l}\infty \\
0 \\
0\end{array}$ & $\overbrace{0}^{\infty}+]_{0}^{+}$ & \\
\hline 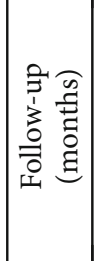 & 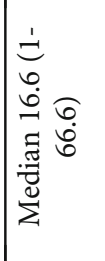 & $\stackrel{ }{\wedge}$ & $\stackrel{\circ}{\circ}$ & 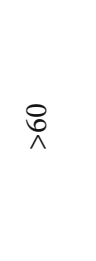 & 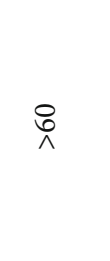 & 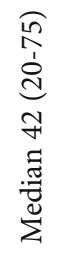 & $\stackrel{8}{\circ}$ & 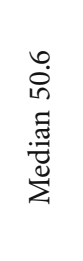 & 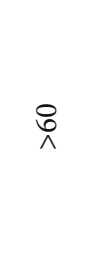 & 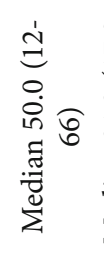 & 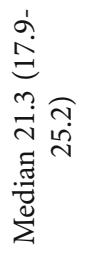 & 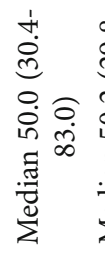 & 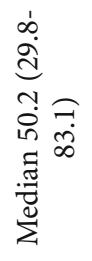 & 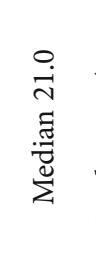 & 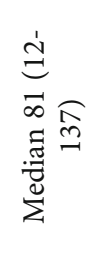 & 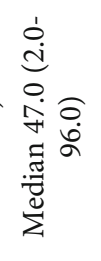 & : & & \\
\hline $\begin{array}{l}\text { : } \\
\text { 訁ँ }\end{array}$ & $\begin{array}{l}\text { 总 } \\
\text { ô }\end{array}$ & $\tilde{0}$ & os & o & 0 & 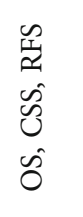 & $\ddot{0}$ & 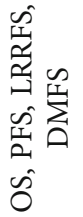 & $\tilde{0}$ & $\begin{array}{l}\text { 崩 } \\
\hat{0} \\
\hat{0}\end{array}$ & 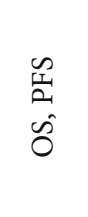 & $\begin{array}{l}\tilde{3} \\
\tilde{o}\end{array}$ & $\begin{array}{l}\tilde{B} \\
\tilde{8}\end{array}$ & $\begin{array}{l}\text { 出 } \\
\text { w } \\
\text { s }\end{array}$ & $\begin{array}{l}\text { s } \\
\text { th } \\
\text { st }\end{array}$ & 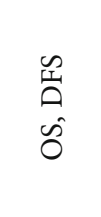 & $\begin{array}{l}\text { 是 } \\
\hat{0} \\
\hat{0}\end{array}$ & 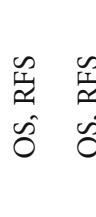 & \\
\hline 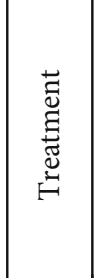 & 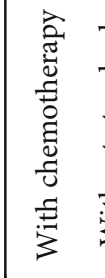 & 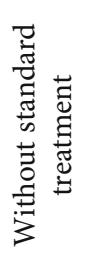 & 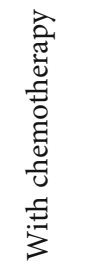 & 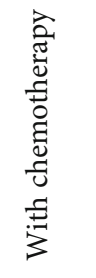 & 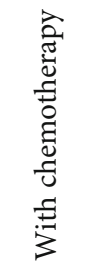 & 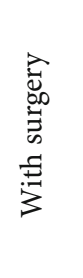 & 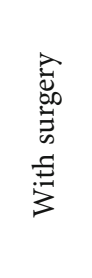 & $\overrightarrow{\frac{\mathscr{x}}{2}}$ & 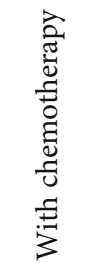 & 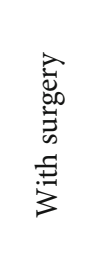 & 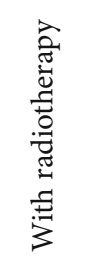 & 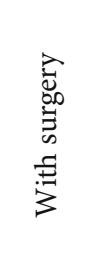 & 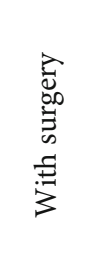 & 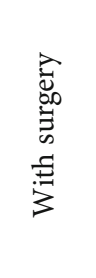 & 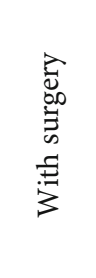 & 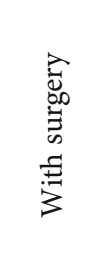 & 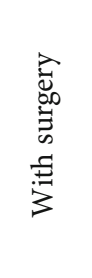 & 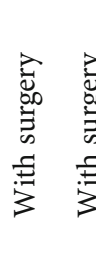 & \\
\hline 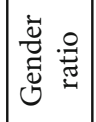 & $\begin{array}{l}0 \\
\frac{0}{2} \\
\end{array}$ & 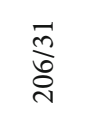 & 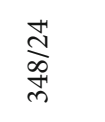 & 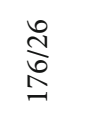 & $\stackrel{\curvearrowright}{\hat{\aleph}}$ & 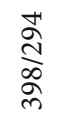 & $\frac{0}{\frac{0}{0}}$ & 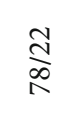 & 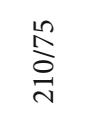 & $\underset{\substack{\text { f } \\
\text { f }}}{\text { for }}$ & 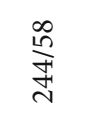 & 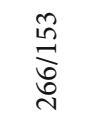 & $\underset{\underset{ユ}{N}}{\stackrel{N}{A}}$ & 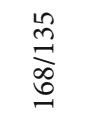 & 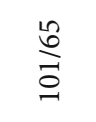 & 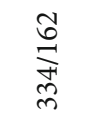 & $\stackrel{\infty}{\underset{\exists}{\exists}}$ & 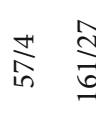 & \\
\hline 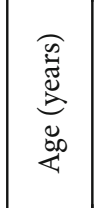 & $\mid$ & 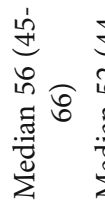 & 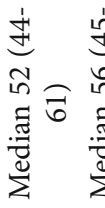 & 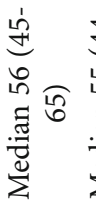 & 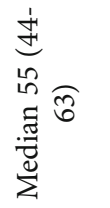 & 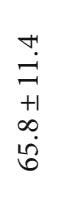 & $\overleftrightarrow{z}$ & $\overleftrightarrow{z}$ & 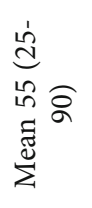 & $\begin{array}{l}0 \\
0 \\
+1 \\
0 \\
\dot{0} \\
0\end{array}$ & 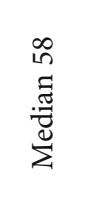 & $\begin{array}{l}\vec{j} \\
\overrightarrow{\mathrm{d}} \\
+1 \\
\dot{+} \\
\overrightarrow{0}\end{array}$ & $\begin{array}{l}\stackrel{1}{I} \\
+ \\
+ \\
\text { i }\end{array}$ & 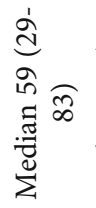 & 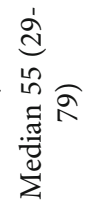 & 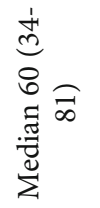 & 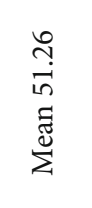 & 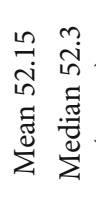 & ત્d \\
\hline 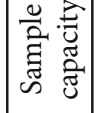 & वे & $\widehat{\approx}$ & 於 & ๙ิ & $\infty$ & ठิ & 呆 & 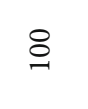 & ম্রి & ః̊ & $\tilde{I}$ & $\vec{F}$ & 荢 & ి్̊లి & శ్ & $\stackrel{+}{\circ}$ & & $\vec{\sigma} \infty_{\infty}^{\infty}$ & \\
\hline త్ & 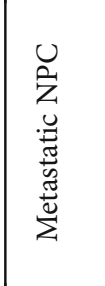 & 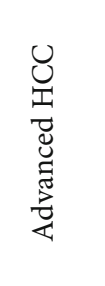 & & 雚 & & $\begin{array}{l}\text { D } \\
\text { D }\end{array}$ & 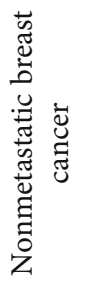 & 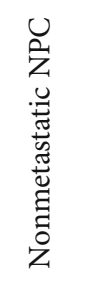 & 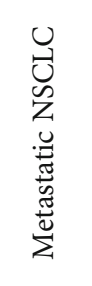 & 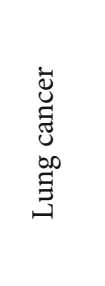 & 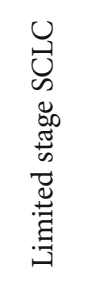 & 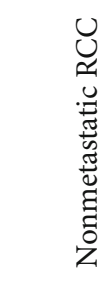 & 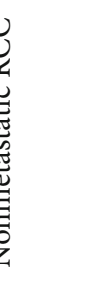 & J্ট & 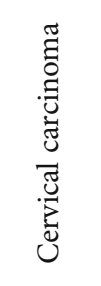 & $\begin{array}{l}u \\
\text { U. } \\
\text { z }\end{array}$ & ن & Uي & \\
\hline 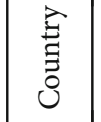 & 苟 & 䋃 & & 苞 & & 莺 & 䄆 & 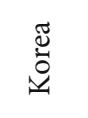 & 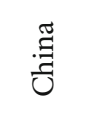 & 苞 & 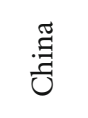 & : & j & 苟 & 苞 & 蚫 & 苞 & & \\
\hline 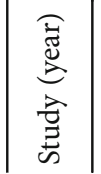 & 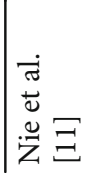 & 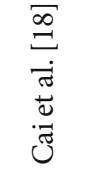 & & 苛 & & & & & 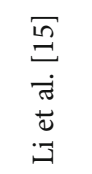 & 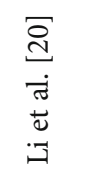 & 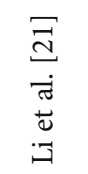 & 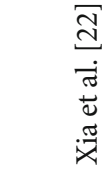 & & 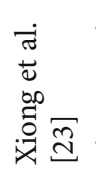 & 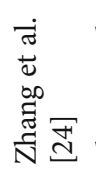 & 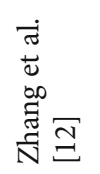 & & 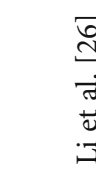 & \\
\hline
\end{tabular}




\begin{tabular}{|c|c|c|c|}
\hline Study & & $\mathrm{HR}(95 \% \mathrm{Cl})$ & Weight \% \\
\hline Nie (2017) & $\frac{1}{1} \cdot$ & $3.27(1.71,6.25)$ & 4.27 \\
\hline Cai (2018) & $\rightarrow$ & $1.69(1.15,2.47)$ & 7.45 \\
\hline Chen (2018)-1 & 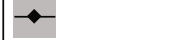 & $1.57(1.17,2.12)$ & 8.74 \\
\hline Chen (2018)-2 & 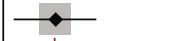 & $2.14(1.17,3.91)$ & 4.68 \\
\hline Chen (2018)-3 & 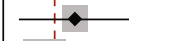 & $2.87(1.25,6.29)$ & 3.14 \\
\hline $\operatorname{Tan}(2018)$ & $\rightarrow$ & $1.82(1.37,2.42)$ & 9.02 \\
\hline Long (2019) & & $2.24(1.02,4.88)$ & 3.30 \\
\hline Kim (2019) & $\rightarrow$ & $1.77(0.74,4.24)$ & 2.78 \\
\hline Li (2019) & $\rightarrow$ & $1.52(1.17,1.98)$ & 9.32 \\
\hline Li (2019) & + & $3.23(1.67,6.25)$ & 4.16 \\
\hline $\operatorname{Li}(2019)$ & $\rightarrow$ & $1.70(1.02,2.78)$ & 5.78 \\
\hline Xia (2019)-1 & $\stackrel{1}{\longrightarrow}$ & $2.74(1.27,5.95)$ & 3.34 \\
\hline Xia (2019)-2 & $1 \longrightarrow$ & $6.01(3.06,11.78)$ & 4.05 \\
\hline Xiong (2019) & 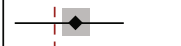 & $2.88(1.19,5.78)$ & 3.24 \\
\hline Zhang (2019) & $\longrightarrow$ & $3.02(1.24,7.41)$ & 2.68 \\
\hline Zhang (2019) & $\rightarrow$ & $1.87(1.22,2.75)$ & 7.07 \\
\hline $\operatorname{Li}(2020)-1$ & $\rightarrow$ & $1.71(1.06,2.76)$ & 6.09 \\
\hline $\operatorname{Li}(2020)-2$ & $\rightarrow$ & $2.44(1.02,5.88)$ & 2.77 \\
\hline $\operatorname{Li}(2020)$ & $\begin{array}{l}1 \\
1\end{array}$ & $27.03(5.38,142.86)$ & 0.94 \\
\hline Zhou (2020) & $\rightarrow$ & $1.65(1.11,2.46)$ & 7.18 \\
\hline Overall $\left(I^{2}=48.1 \%, P=0.009\right)$ & 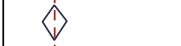 & $2.12(1.80,2.50)$ & 100.00 \\
\hline Note: weights are from random effects analysis & & & \\
\hline $\begin{array}{c} \\
0.007\end{array}$ & & & \\
\hline
\end{tabular}

FIGURE 2: Forest plot for the association between pretreatment AAPR and OS. Abbreviations: OS: overall survival; AAPR: albumin-to-alkaline phosphatase ratio.

comparison studies and literature types were abstracts, letters, editorials, reviews, expert opinions, or case reports.

2.3. Data Extraction and Quality Assessment. Two investigators (Hailun Xie and Lishuang Wei) independently extracted the data and evaluated the quality of all eligible studies. The extracted basic information included the name of the first author, publication year, country, types of cancer, sample capacity, patient characteristics (age, male/female percentage), cutoff value of AAPR, treatment methods, outcome, follow-up time, and analysis method. The extraction of prognostic indicators included HR with corresponding 95\% CI. If only the Kaplan-Meier curve provided prognostic outcomes, we used Engauge Digitizer 4.1 software to obtain the estimated HR using the Tierney method [16]. The quality of the included studies was evaluated referring to the Newcastle-Ottawa Scale (NOS) [17]. The NOS score ranged from 0 to 9 points, which included three aspects: patient selection ( $0-4$ points), comparability ( $0-2$ points), and outcome (0-3 points). Studies with NOS scores of $\geq 6$ were considered as high quality.

2.4. Statistical Analysis. Data analysis was performed by Stata version 13.0 (STATA Corporation, College Station, TX, USA). A pooled HR with 95\% CI was calculated. Cochran's $Q$ test and Higgins $I^{2}$ statistics were used to evaluate the heterogeneity among the studies. The random effects model was applied when there was significant heterogeneity $\left(I^{2}>50 \%\right.$ or $\left.P<0.10\right)$. Otherwise, the fixed effects model was applied. Subgroup analysis and metaregression analysis were used to assess the sources of heterogeneity. Sensitivity analysis was used to evaluate the reliability of the results by recalculating the pooled HR with 95\% CI after deleting one study at a time. Begg's funnel plot was used to assess publication bias. In this study, $P<0.05$ was considered statistically significant.

\section{Results}

3.1. Study Characteristics. A total of 229 published articles were initially retrieved based on the PRISMA guidelines. After deleting duplicate articles and unrelated literature, 141 articles required further evaluation. After the title and abstract screening, 123 articles were excluded for the following reasons: not about AAPR with cancer $(n=72)$ and conference abstracts $(n=51)$. We downloaded 18 full-text articles to further evaluate whether they could be included in our meta-analysis. One article was excluded because it did not have a single cutoff value, and another was excluded because it did not report prognosis. Finally, 16 articles involving 5,716 cases were included in our meta-analysis [10, 12-15, 18-27]. The flow chart of document retrieval is illustrated in Figure 1. Among the included articles, 15 were from China and one from South Korea. All included studies were retrospectively designed. Sixteen articles contained 20 cohort studies, among which one contained three cohort studies, and two contained two cohort studies. The publication year was between 2017 and 
TABLE 2: Stratification analysis for the meta-analysis with overall survival (OS) in patients with cancer.

\begin{tabular}{|c|c|c|c|c|c|c|}
\hline \multirow{2}{*}{ Subgroup } & \multirow{2}{*}{ No. of cohorts } & \multirow{2}{*}{ No. of patients } & \multirow{2}{*}{ Pooled HR (95\% CI) } & \multirow{2}{*}{$P$} & \multicolumn{2}{|c|}{ Heterogeneity } \\
\hline & & & & & $I^{2}(\%)$ & $P_{\mathrm{h}}$ \\
\hline Altogether & 20 & 5716 & $2.12(1.80-2.94)$ & $<0.001$ & 48.1 & 0.009 \\
\hline \multicolumn{7}{|l|}{ Publishing time } \\
\hline$<2019$ & 6 & 1794 & $1.86(1.55-2.23)$ & $<0.001$ & 11.9 & 0.339 \\
\hline$\geq 2019$ & 14 & 3922 & $2.30(1.80-2.94)$ & $<0.001$ & 57.3 & 0.004 \\
\hline \multicolumn{7}{|l|}{ Country } \\
\hline China & 19 & 5616 & $2.14(1.80-2.54)$ & $<0.001$ & 50.8 & 0.006 \\
\hline Korea & 1 & 100 & $1.77(0.74-4.24)$ & NA & NA & NA \\
\hline \multicolumn{7}{|l|}{ Sample capacity } \\
\hline$<230$ & 10 & 1541 & $2.52(1.80-3.53)$ & $<0.001$ & 62.2 & 0.005 \\
\hline$\geq 230$ & 11 & 4175 & $1.81(1.58-2.07)$ & $<0.001$ & 5.7 & 0.388 \\
\hline \multicolumn{7}{|l|}{ Cutoff value } \\
\hline$<0.5$ & 14 & 3040 & $2.22(1.76-2.81)$ & $<0.001$ & 60.2 & 0.002 \\
\hline$\geq 0.5$ & 6 & 2676 & $1.97(1.63-2.38)$ & $<0.001$ & 0 & 0.575 \\
\hline \multicolumn{7}{|l|}{ Cancer system } \\
\hline Digestive cancer & 8 & 1594 & $2.13(1.57-2.87)$ & $<0.001$ & 52.2 & 0.041 \\
\hline Respiratory cancer & 7 & 1831 & $1.86(1.51-2.28)$ & $<0.001$ & 25.6 & 0.234 \\
\hline Urinary cancer & 3 & 1315 & $2.98(1.41-6.32)$ & 0.004 & 81 & 0.005 \\
\hline Breast cancer & 1 & 746 & $2.24(1.03-4.88)$ & NA & NA & NA \\
\hline Gynecological cancers & 1 & 230 & $3.02(1.23-7.40)$ & NA & NA & NA \\
\hline \multicolumn{7}{|l|}{ Primary therapy } \\
\hline With chemotherapy & 5 & 1155 & $1.88(1.44-2.45)$ & $<0.001$ & 42.6 & 0.137 \\
\hline With radiotherapy & 1 & 122 & $1.70(1.03-2.80)$ & NA & NA & NA \\
\hline With surgery & 11 & 3878 & $2.64(1.97-3.54)$ & $<0.001$ & 57.3 & 0.009 \\
\hline Mixed & 2 & 324 & $1.67(1.16-2.40)$ & 0.006 & 0 & 0.889 \\
\hline Without treatment & 1 & 237 & $1.69(1.16-2.47)$ & NA & NA & NA \\
\hline \multicolumn{7}{|l|}{ Analytic method } \\
\hline Univariate & 3 & 387 & $2.88(1.28-6.48)$ & 0.011 & 77.2 & 0.013 \\
\hline Multivariate & 17 & 5329 & $1.99(1.70-2.32)$ & $<0.001$ & 35.4 & 0.074 \\
\hline
\end{tabular}

2020. The sample capacity ranged from 61 to 692 , and the cutoff of AAPR varied from 0.35 to 0.68 . This study involved a variety of cancers, including NPC, HCC, UTUC, breast cancer, NSCLC, lung cancer, SCLC, cholangiocarcinoma (CCA), cervical carcinoma, and extensivedisease SCLC (ED-SCLC). In terms of the quality evaluation of the selected cohort studies, the NOS score of 16 cohort studies was 8 , the NOS score of one cohort study was 7 , and the NOS score of three cohort studies was 6 . The baseline information is shown in Table 1 .

3.2. Association between Pretreatment AAPR and OS. Twenty cohort studies enrolling 5,716 cases reported the prognostic significance of pretreatment AAPR for OS in cancer. Comprehensive results indicated that low pretreatment AAPR was significantly associated with poor OS, compared with high pretreatment AAPR $(\mathrm{HR}=2.12,95 \% \mathrm{CI}=1.80-2.50$, $P<0.001)$. A random effects model was applied due to the obvious heterogeneity $\left(I^{2}=48.1 \%, P=0.009\right)$ (Figure 2$)$. The meta-analysis for OS included more than 10 studies, and heterogeneity was found in the results. Therefore, we conducted a subgroup analysis of publication year, country, sample capacity, cutoff value, cancer system, primary therapy, and analytic methods (Table 2). The results still indicated that low pretreatment AAPR had an adverse effect on OS in cancer patients. At the same time, when classified by these factors, heterogeneity was eliminated in some subgroup meta-analyses, such as publication year $<2019$, sample capacity $\geq 230$, cutoff value $\geq 0.5$, respiratory cancer, chemotherapy, and mixed therapy subgroups. To explore further the impact of different subgroups on pooled HR, we conducted a meta-regression analysis. The influence of these different subgroups on pooled $\mathrm{HR}$ was not significant $\left(P_{\text {study design }}=0.763, \quad P_{\text {country }}=0.854, \quad P_{\text {sample capacity }}=0.608\right.$, $P_{\text {cutoff value }}=0.608, P_{\text {cancer system }}=0.744, P_{\text {primary therapy }}=0.114$ , and $P_{\text {analytic method }}=0.433$ ).

3.3. Sensitivity Analysis for OS. Sensitivity analysis was used to assess the potential impact of the individual studies on the comprehensive results. We performed a sensitivity analysis by recalculating the pooled HR with 95\% CI after deleting one study at a time (Figure 3 ). The results showed that omitting any included studies did not change the effect of 


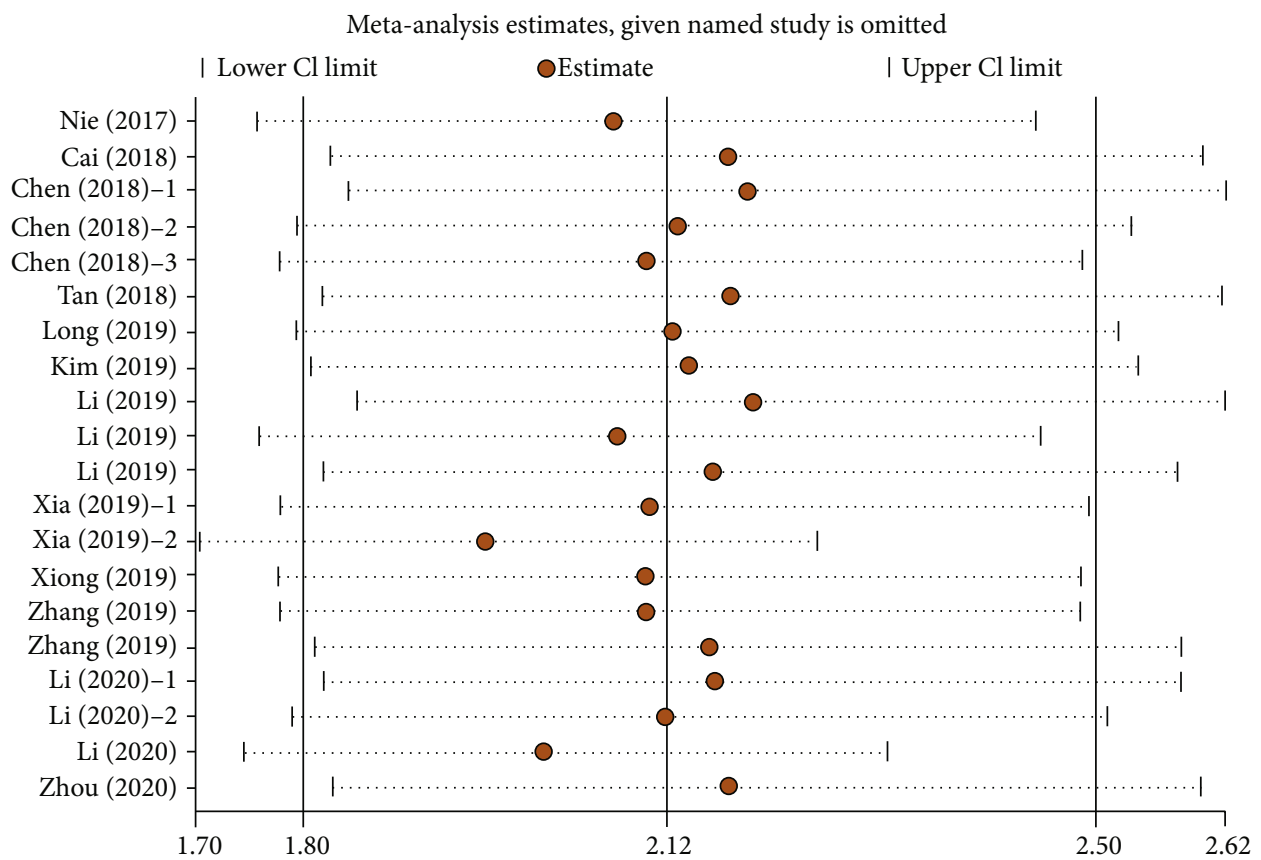

FIGURE 3: Sensitivity analysis for the association between pretreatment AAPR and OS. OS: overall survival.

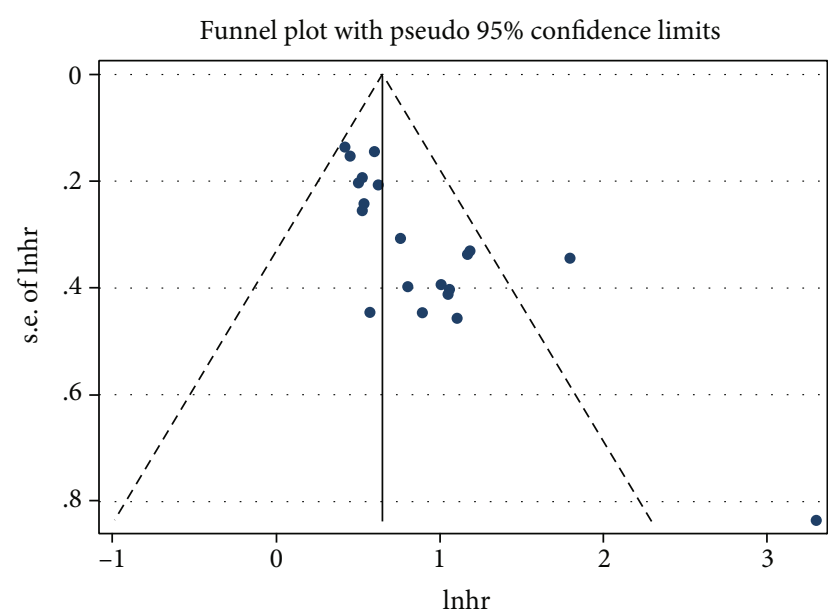

Figure 4: Begg's funnel plot for the assessment of potential publication bias according to OS. Abbreviations: OS: overall survival.

pretreatment AAPR on the comprehensive meta-analysis of OS. In other words, the comprehensive result of our metaanalysis was stable.

3.4. Publication Bias for OS. Begg's funnel plots were used to evaluate the potential publication bias (Figure 4). The funnel plot showed that the included studies presented asymmetry and the $P$ value was $<0.05$, suggesting that there was potential publication bias in the meta-analysis for OS.

3.5. Association between Pretreatment AAPR and Other Outcomes. We studied the prognostic effect of pretreatment AAPR on CSS, DFS, PFS, RFS, DMFS, and LRRFS of cancer patients (Figure 5). Three cohort studies including 1,315 cases reported the prognostic value of pretreatment AAPR for CSS (Figure 5(a)). A random effects model $\left(I^{2}=52.9 \%\right.$, $P=0.119$ ) was used because of the obvious heterogeneity. The combined results showed that patients with low pretreatment AAPR had poorer CSS than those with high pretreatment AAPR $(\mathrm{HR}=2.89,95 \%$ CI: $1.46-5.71, P<0.001)$. Three cohort studies consisted of 1,116 patients reported HR for DFS (Figure 5(b)). The heterogeneity test indicated that there was no obvious heterogeneity, and the fixed effects model was adopted $\left(I^{2}=0.0 \%, P=0.629\right)$. The pooled HR with 95\% CI was 1.91 (95\% CI: $1.43-2.53, P<0.001$ ), indicating a significant relationship between low pretreatment AAPR levels and poor DFS in cancer. Four cohort studies including 734 cases reported the prognostic value of pretreatment AAPR for PFS (Figure 5(c)). A fixed effects model $\left(I^{2}=0.0 \%, P=0.533\right)$ was used. The results showed that patients with low pretreatment AAPR had worse PFS compared to patients with high pretreatment AAPR $(\mathrm{HR}=1.93$, 95\% CI: $1.49-2.52, P<0.001)$. However, there was no statistical relationship between pretreatment AAPR and RFS $(\mathrm{HR}=2.08,95 \%$ CI: $0.93-4.66, P=0.076)$ (Figure 5(d)), DMFS $\quad(\mathrm{HR}=0.62, \quad 95 \% \quad$ CI: $\quad 0.14-2.76, \quad P=0.534)$ (Figure 5(e)), or LRRFS (HR $=2.67,95 \%$ CI: $0.78-9.15, P=$ 0.117) (Figure 5(f)).

\section{Discussion}

AAPR, composed of albumin and alkaline phosphatase, was initially reported to be associated with poor prognosis of patients with HCC in 2015 [28]. Since then, many studies have demonstrated that pretreatment AAPR is a useful prognostic indicator for a variety of cancers. Pretreatment AAPR is expected to be a simple and effective tool for predicting the clinical outcome of cancer patients. However, the underlying mechanism of how pretreatment AAPR affects the prognosis of cancer remains unclear. As a composite indicator based on 


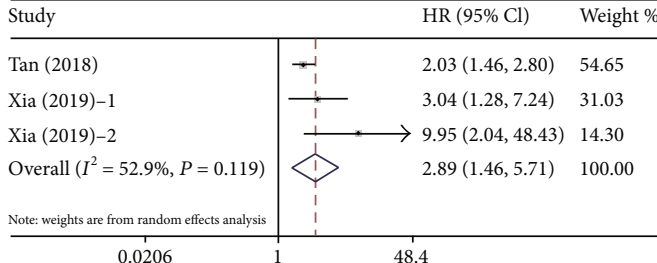

(a)

\begin{tabular}{|c|c|c|c|}
\hline Study & & $\mathrm{HR}(95 \% \mathrm{Cl})$ & Weight $\%$ \\
\hline Nie (2017) & $\frac{1}{1}$ & $-2.30(1.22,4.33)$ & 17.24 \\
\hline Kim (2019) & & $1.40(0.63,3.11)$ & 10.91 \\
\hline Li (2019) & & $1.61(1.01,2.50)$ & 33.81 \\
\hline Xiong (2019) & & $2.31(1.40,3.29)$ & 38.04 \\
\hline Overall $\left(I^{2}=0 \%, P=0.533\right)$ & $\diamond$ & $1.93(1.49,2.52)$ & 100.00 \\
\hline
\end{tabular}

(c)

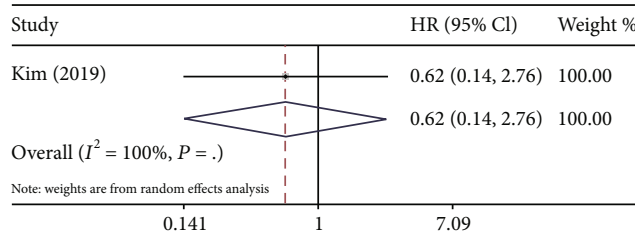

(e)

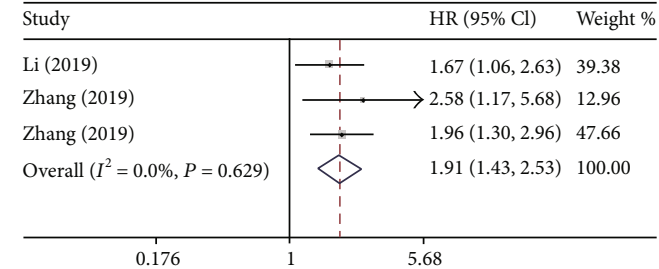

(b)

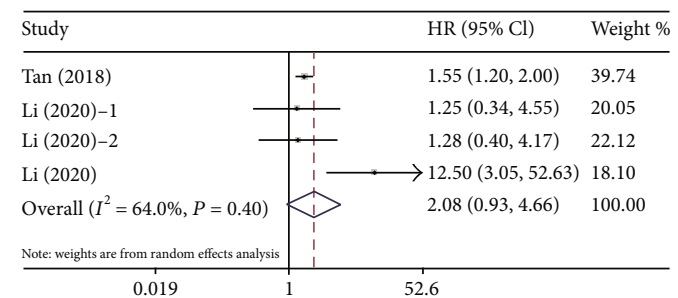

(d)

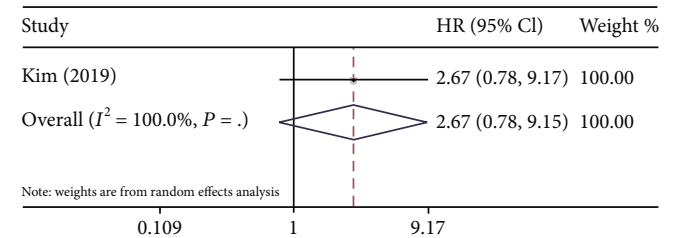

(f)

FIGURE 5: Forest plot for the association between pretreatment AAPR and other outcomes. (a) Forest plot for CSS. (b) Forest plot for DFS. (c) Forest plot for PFS. (d) Forest plot for RFS. (e) Forest plot for DMFS. (f) Forest plot for LRRFS. Abbreviations: CSS: cancer-specific survival; DFS: disease-free survival; PFS: progression-free survival; RFS: recurrence-free survival; DMFS: distant metastasis-free survival (DMFS); and LRRFS: locoregional relapse-free survival (LRRFS).

albumin and alkaline phosphatase, the prognostic value of pretreatment AAPR in cancer could be elucidated by investigating the function of its components (albumin and alkaline phosphatase). Albumin is a functional serum protein. It is an important clinical indicator that reflects the nutritional status and liver synthesis ability, and it has also been proved to regulate the inflammatory response throughout the body and play an antioxidant role in tumorigenesis [29]. Additionally, low albumin may affect the metabolism and function of immune cells, which may reduce immune function and cause adverse anticancer reactions [30]. Recently, many studies have shown that albumin is a useful prognostic predictor in various malignancies such as UTUC [31], HCC [32], and prostate cancer [33]. Alkaline phosphatase, a hydrolytic enzyme involved in biological processes, such as epithelial mesenchymal transformation and ERK1/2 dephosphorylation $[34,35]$, can cause the cessation of inflammatory signaling and induce an inhibitory immune response by regulating purinergic signaling. Alkaline phosphatase is mainly found in the liver, bone, and kidney. Alkaline phosphatase may be elevated in certain conditions like the liver, bone, and kidney diseases (malignant and benign) and especially in cholestasis. However, it is worth noting that Li et al. [15] showed that alkaline phosphatase has a pleiotropic effect in tumor progression, and it has prognostic value in cancer patients regardless of whether there is liver or bone metastasis. It has been confirmed to be elevated in various cancers and be associated with poor prognosis, including colorectal cancer, gastric cancer, and esophageal carcinoma [36-38]. Therefore, pretreatment with AAPR may be a more objective tumor marker that comprehensively reflects the balance between nutritional status and cancer-related inflammation.

Sixteen articles including 5,716 patients were included in our meta-analysis. In the meta-analysis of primary outcome, we found that cancer patients with low pretreatment AAPR had worse OS than those with high pretreatment AAPR. Stratified analysis was performed to correct for the influence of different subgroups on the results. The results showed that despite differences in publication year, sample capacity, cutoff value, cancer system, primary therapy, and analytical methods among different populations; the combined results still indicated that low pretreatment AAPR was correlated with poor OS. Further meta-regression analysis showed that the different subgroups did not affect the combined results of meta-analysis. We also conducted a sensitivity analysis by deleting one study at a time, and the results did not significantly change the pooled $\mathrm{HR}$, indicating that our results were reliable. We also found a significant association between low pretreatment AAPR and adverse CSS, DFS and PFS in cancer patients. However, there was no statistical association between low pretreatment AAPR and adverse RFS, DMFS, and LRFRS. In summary, our meta-analysis confirmed that pretreatment AAPR can be a powerful predictor of poor outcomes in cancer patients. Although Guo et al. [39] conducted a meta-analysis on AAPR, our research carried out some new and useful explorations, mainly in the following aspects. Guo 
et al. mainly focused on the relationship between AAPR and OS. In our study, not only did we find that AAPR was associated with the primary outcome (OS) but we also found that AAPR was associated with poor secondary outcomes (CSS, DFS, and PFS), although there was no obvious correlation with outcomes such as RFS, DMFS, or LRFRS. We also conducted a more detailed subgroup analysis and metaregression analysis to explore more comprehensively the value of AAPR in cancer patients. We also updated two studies including three cohorts. We also noted that there were some defects in the study of Guo et al.; for example, the research of Zhang et al. (2019) [24] on cervical carcinoma was not included and research by Chan et al. (2018) [28] showing that there were three categories of AAPR should be excluded. We believe that our study was more comprehensive and more accurate to summarize the relationship between AAPR and prognosis of cancer patients.

However, in view of some limitations, the results of our meta-analysis should be interpreted with caution. First, all included studies were conducted in Asia and were retrospective studies, which are more susceptible to potential selection bias. The current comprehensive conclusions mainly support the prognostic value of AAPR in Asian cancer patients. The practicality of AAPR is yet to be demonstrated in a prospective, multicenter study worldwide. Second, there was obvious heterogeneity in the meta-analysis of OS, but the metaregression analysis showed that the subgroups were not the sources of the heterogeneity. We speculate that the sources of heterogeneity in this study might have been the small total sample size and the number of included studies, which need to be explored further in a large sample and multicenter prospective study. Third, there was publication bias in our study, but the sensitivity analysis showed that the results were reliable. Despite these limitations, we provided a meaningful exploration of the prognostic value of pretreatment AAPR in cancer patients based on available evidence.

\section{Conclusion}

This meta-analysis revealed that low pretreatment AAPR was significantly associated with adverse clinical outcomes in cancer patients. Pretreatment AAPR could be a valuable noninvasive prognostic indicator for cancer patients. Large, multicenter, prospective cohort studies are needed to evaluate further the role of pretreatment AAPR in cancer patients.

\section{Data Availability}

Please contact author for data requests.

\section{Conflicts of Interest}

The authors declared no conflicts of interest.

\section{Authors' Contributions}

Hailun Xie and Lishuang Wei contributed equally to this work.

\section{Supplementary Materials}

Supplemental files 1 PRISMA 2009 checklist. (Supplementary Materials)

\section{References}

[1] W. Chen, K. Sun, R. Zheng et al., "Cancer incidence and mortality in China, 2014," Chinese Journal of Cancer Research, vol. 30, no. 1, pp. 1-12, 2018.

[2] F. I. Bray, J. Ferlay, I. Soerjomataram, R. L. Siegel, L. A. Torre, and A. Jemal, "Global cancer statistics 2018: GLOBOCAN estimates of incidence and mortality worldwide for 36 cancers in 185 countries," CA: A Cancer Journal for Clinicians, vol. 68, no. 6, pp. 394-424, 2018.

[3] A. Mantovani, P. Allavena, A. Sica, and F. R. Balkwill, "Cancerrelated inflammation," Nature, vol. 454, no. 7203, pp. 436-444, 2008.

[4] F. R. Balkwill and A. Mantovani, "Inflammation and cancer: back to Virchow?" The Lancet, vol. 357, no. 9255, pp. 539545, 2001.

[5] E. Van Cutsem and J. Arends, "The causes and consequences of cancer-associated malnutrition," European Journal of Oncology Nursing, vol. 9, no. 9, 2005.

[6] D. C. Mcmillan, "The systemic inflammation-based Glasgow prognostic score: a decade of experience in patients with cancer," Cancer Treatment Reviews, vol. 39, no. 5, pp. 534-540, 2013.

[7] M. J. Proctor, D. S. Morrison, D. Talwar et al., “An inflammation-based prognostic score (mGPS) predicts cancer survival independent of tumour site: a Glasgow Inflammation Outcome Study," British Journal of Cancer, vol. 104, no. 4, pp. 726-734, 2011.

[8] Y. Liu, S. Chen, C. Zheng et al., "The prognostic value of the preoperative c-reactive protein/albumin ratio in ovarian cancer," BMC Cancer, vol. 17, no. 1, 2017.

[9] S. Tang, H. Xie, J. Kuang, F. Gao, J. Gan, and H. Ou, "The value of geriatric nutritional risk index in evaluating postoperative complication risk and long-term prognosis in elderly colorectal cancer patients," Cancer Management and Research, vol. Volume 12, pp. 165-175, 2020.

[10] Z.-H. Chen, X.-P. Zhang, X.-R. Cai et al., "The predictive value of albumin-to-alkaline phosphatase ratio for overall survival of hepatocellular carcinoma patients treated with trans-catheter arterial chemoembolization therapy," Journal of Cancer, vol. 9, no. 19, pp. 3467-3478, 2018.

[11] M. Nie, P. Sun, C. Chen et al., "Albumin-to-alkaline phosphatase ratio: a novel prognostic index of overall survival in cisplatin-based chemotherapy-treated patients with metastatic nasopharyngeal carcinoma," Journal of Cancer, vol. 8, no. 5, pp. 809-815, 2017.

[12] L. Zhang, H. Zhang, D. Yue et al., "The prognostic value of the preoperative albumin to alkaline phosphatase ratio in patients with non-small cell lung cancer after surgery," Thoracic Cancer, vol. 10, no. 7, pp. 1581-1589, 2019.

[13] P. Tan, N. Xie, J. Ai et al., "The prognostic significance of albumin-to-alkaline phosphatase ratio in upper tract urothelial carcinoma," Scientific Reports, vol. 8, no. 1, p. 12311, 2018

[14] Z.-Q. Long, X. Hua, W.-W. Zhang et al., "Prognostic impact of the pretreatment albumin to alkaline phosphatase ratio for nonmetastatic breast cancer patients," Cancer Management and Research, vol. 11, pp. 4809-4814, 2019. 
[15] X. Li, B. Li, H. Zeng et al., "Prognostic value of dynamic albumin-to-alkaline phosphatase ratio in limited stage smallcell lung cancer," Future Oncology, vol. 15, no. 9, pp. 9951006, 2019.

[16] J. F. Tierney, L. A. Stewart, D. Ghersi, S. Burdett, and M. R. Sydes, "Practical methods for incorporating summary timeto-event data into meta-analysis," Trials, vol. 8, no. 1, 2007.

[17] A. Stang, "Critical evaluation of the Newcastle-Ottawa scale for the assessment of the quality of nonrandomized studies in meta-analyses," European Journal of Epidemiology, vol. 25, no. 9, pp. 603-605, 2010.

[18] X. Cai, Z. Chen, J. Chen et al., “Albumin-to-alkaline phosphatase ratio as an independent prognostic factor for overall survival of advanced hepatocellular carcinoma patients without receiving standard anti-cancer therapies," Journal of Cancer, vol. 9, no. 1, pp. 189-197, 2018.

[19] J. S. Kim, B. Keam, D. S. Heo et al., "The prognostic value of albumin-to-alkaline phosphatase ratio before radical radiotherapy in patients with non-metastatic nasopharyngeal carcinoma: a propensity score matching analysis," Cancer Research and Treatment, vol. 51, no. 4, pp. 1313-1323, 2019.

[20] D. Li, H. Yu, and W. Li, “Albumin-to-alkaline phosphatase ratio at diagnosis predicts survival in patients with metastatic non-small-cell lung cancer," Oncotargets and Therapy, vol. Volume 12, pp. 5241-5249, 2019.

[21] S.-J. Li, W.-Y. Lv, H. Du et al., "Albumin-to-alkaline phosphatase ratio as a novel prognostic indicator for patients undergoing minimally invasive lung cancer surgery: propensity score matching analysis using a prospective database," International Journal of Surgery, vol. 69, pp. 32-42, 2019.

[22] A. Xia, Y. Chen, J. Chen, Y. Pan, L. Bao, and X. Gao, "Prognostic value of the albumin-to-alkaline phosphatase ratio on urologic outcomes in patients with non-metastatic renal cell carcinoma following curative nephrectomy," Journal of Cancer, vol. 10, no. 22, pp. 5494-5503, 2019.

[23] J. P. Xiong, J. Y. Long, W. Y. Xu et al., “Albumin-to-alkaline phosphatase ratio: a novel prognostic index of overall survival in cholangiocarcinoma patients after surgery," World Journal of Gastrointestinal Oncology, vol. 11, no. 1, pp. 39-47, 2019.

[24] C. Zhang, Y. Li, R. Ji et al., "The prognostic significance of pretreatment albumin/alkaline phosphatase ratio in patients with stage IB-IIA cervical cancer," OncoTargets and Therapy, vol. Volume 12, pp. 9559-9568, 2019.

[25] H. Li, L. Wang, L. Chen et al., "Prognostic value of albumin-toalkaline phosphatase ratio in hepatocellular carcinoma patients treated with liver transplantation," Journal of Cancer, vol. 11, no. 8, pp. 2171-2180, 2020.

[26] Q. Li, Z. Lyu, L. Wang, F. Li, Z. Yang, and W. Ren, “Albuminto-alkaline phosphatase ratio associates with good prognosis of hepatitis B virus-positive HCC patients," OncoTargets and Therapy, vol. Volume 13, pp. 2377-2384, 2020.

[27] S. Zhou, H. Wang, W. Jiang, Q. Yu, and A. Zeng, "Prognostic value of pretreatment albumin-to-alkaline phosphatase ratio in extensive-disease small-cell lung cancer: a retrospective cohort study," Cancer Management and Research, vol. Volume 12, pp. 2015-2024, 2020.

[28] A. W. H. Chan, S. L. Chan, F. K. F. Mo et al., "Albumin-toalkaline phosphatase ratio: a novel prognostic index for hepatocellular carcinoma.," Disease Markers, vol. 2015, Article ID 564057, 10 pages, 2015.
[29] V. Arroyo, R. García-Martinez, and X. Salvatella, "Human serum albumin, systemic inflammation, and cirrhosis," Journal of Hepatology, vol. 61, no. 2, pp. 396-407, 2014.

[30] N. Ahluwalia, “Aging, nutrition and immune function," Journal of Nutrition Health \& Aging, vol. 8, no. 1, pp. 2-6, 2004.

[31] J. H. Ku, M. Kim, W. S. Choi, C. Kwak, and H. H. Kim, "Preoperative serum albumin as a prognostic factor in patients with upper urinary tract urothelial carcinoma," International Brazilian Journal of Urology, vol. 40, no. 6, pp. 753-762, 2014.

[32] A. Hiraoka, T. Kumada, K. Nouso et al., "Proposed new subgrouping for intermediate-stage hepatocellular carcinoma using albumin-bilirubin grade," Oncology, vol. 91, no. 3, pp. 153-161, 2016.

[33] K. N. Chi, T. Kheoh, C. J. Ryan et al., "A prognostic index model for predicting overall survival in patients with metastatic castration-resistant prostate cancer treated with abiraterone acetate after docetaxel," Annals of Oncology, vol. 27, no. 3, pp. 454-460, 2016.

[34] S. R. Rao, A. E. Snaith, D. Marino et al., “Tumour-derived alkaline phosphatase regulates tumour growth, epithelial plasticity and disease-free survival in metastatic prostate cancer," British Journal of Cancer, vol. 116, no. 2, pp. 227-236, 2017.

[35] U. Sharma, D. Pal, and R. Prasad, "A novel role of alkaline phosphatase in the ERK1/2 dephosphorylation in renal cell carcinoma cell lines: a new plausible therapeutic target," Biochimie, vol. 107, Part B, pp. 406-409, 2014.

[36] H.-Y. Hung, J.-S. Chen, R. T. Chien-YuhYeh et al., "Preoperative alkaline phosphatase elevation was associated with poor survival in colorectal cancer patients," International Journal of Colorectal Disease, vol. 32, no. 12, pp. 1775-1778, 2017.

[37] A. Aminian, F. Karimian, R. Mirsharifi et al., "Correlation of serum alkaline phosphatase with clinicopathological characteristics of patients with oesophageal cancer," Eastern Mediterranean Health Journal, vol. 17, no. 11, pp. 862-866, 2011.

[38] T. Namikawa, N. Ishida, S. Tsuda et al., "Prognostic significance of serum alkaline phosphatase and lactate dehydrogenase levels in patients with unresectable advanced gastric cancer," Gastric Cancer, vol. 22, no. 4, pp. 684-691, 2019.

[39] X. Guo, Q. Zou, J. Yan, X. Zhen, and H. Gu, "Prognostic effect of pretreatment albumin-to-alkaline phosphatase ratio in human cancers: a meta-analysis," PLoS One, vol. 15, no. 8, article e0237793, 2020. 